\title{
Croissance et diversité des attentes
}

L'enseignement supérieur en Inde

Growth and the diversity of expectations. Higher Education in India

Expansión y diversidad de expectativas. La enseñanza superior en India

\section{Ved Prakash}

Traducteur : Robert Elbaz

\section{OpenEdition}

\section{Journals}

Édition électronique

URL : http://journals.openedition.org/ries/95

DOI : $10.4000 /$ ries.95

ISSN : 2261-4265

Éditeur

Centre international d'études pédagogiques

Édition imprimée

Date de publication : 1 septembre 2007

Pagination : 27-38

ISBN : 978-2-85420-570-

ISSN : $1254-4590$

\section{Référence électronique}

Ved Prakash, «Croissance et diversité des attentes », Revue internationale d'éducation de Sèvres [En ligne], 45 | septembre 2007, mis en ligne le 22 juin 2011, consulté le 01 mai 2019. URL : http:// journals.openedition.org/ries/95; DOI : 10.4000/ries.95 


\title{
Croissance et diversité des attentes
}

\section{L'enseignement supérieur en Inde*}

\author{
Ved Prakash
}

Les pays du monde entier ont reconnu le rôle décisif de l'enseignement dans l'élaboration d'une économie fondée sur le savoir, pour suivre le rythme des changements que cette économie induit dans le contexte international. L'Inde, l'une des économies dont la croissance est la plus élevée au monde, a elle aussi entrepris de construire une société fondée sur ces bases. Aujourd'hui, le pays dispose du plus grand système d'enseignement supérieur au monde en termes de nombre d'établissements, à savoir 17973 (348 universités et 17625 établissements spécialisés), et du troisième plus grand système d'enseignement supérieur en termes d'effectifs. Cependant, la proportion d'Indiens dans l'enseignement tertiaire est actuellement la plus faible parmi les économies du BRIC - Brésil, Russie, Inde et Chine - (Carnoy, 2006), ce qui ne manque pas de constituer un sujet d'inquiétude. La progression de l'accès à l'enseignement supérieur en Inde a été freinée par le faible taux de réussite en fin d'enseignement secondaire. En fait, l'enseignement secondaire dans le pays a été virtuellement pris en tenaille entre la priorité élevée donnée à l'enseignement primaire et le débat en cours sur la réduction du rôle de l'État dans le développement des enseignements les plus professionnalisants.

Depuis le changement important qui s'est produit dans la politique économique au début des années 1990, lorsqu'elle a mis en œuvre des programmes structurels d'ajustement et a ouvert son économie, l'Inde a cherché à suivre une politique de "sauts de mouton", en ce sens qu'elle devait renoncer à sa tentative d'industrialisation pour poursuivre et encourager l'utilisation de stratégies relatives aux technologies de l'information et des communications (TIC) afin d'achever sa métamorphose vers une économie fondée sur le savoir. Compte tenu de la politique indienne de promotion des hautes technologies et de la demande sans cesse croissante de main-d'œuvre afin de pourvoir des emplois intellectuels hautement qualifiés, la question est maintenant de savoir si le pays est parvenu à mener à bien la construction de cette société fondée sur le savoir, afin d'acquérir un avantage décisif dans l'économie de marché mondiale.

\footnotetext{
*Article traduit par Robert Elbaz.
} 


\section{ENTRE TRADITION ET DÉVELOPPEMENT}

Le système indien de l'enseignement supérieur a une histoire longue et complexe qui remonte à l'ancienne période védique. Initialement, il s'agissait d'un système de transfert de connaissances et d'aptitudes d'une génération à une autre au travers de traditions écrites et orales. L'enseignement supérieur n'était accessible qu'à une élite, en particulier ceux qui occupaient la position la plus élevée dans la hiérarchie sociale, les Brahmanes et autres membres des castes supérieures.

L'instauration du modèle européen d'enseignement supérieur a débuté durant la période coloniale. Le taux de croissance des établissements d'enseignement supérieur fut très faible entre 1857 et 1847, date de l'indépendance. À cette époque précise, le pays disposait d'un système d'enseignement supérieur rudimentaire qui ne comptait que vingt universités et cinq cents établissements spécialisés. Les effectifs dans ces établissements avoisinaient dix mille personnes et il y avait une grande disparité dans la façon dont ces établissements étaient géographiquement distribués. La montée en puissance du développement de l'université se faisait principalement sentir dans les sciences humaines, alors que l'enseignement scientifique ne jouissait ni du même engouement, ni des mêmes moyens.

Après l'indépendance, le système d'enseignement supérieur fut l'objet d'un changement significatif, passant d'un système élitiste à un système de masse. La politique de mise en place d'une économie autosuffisante exigeait une montée en puissance rapide du système d'enseignement supérieur selon les principes de justice distributive, d'équité et d'efficacité. Les trente premières années furent celles de l'expansion des établissements supérieurs publics, expansion dont la priorité absolue était la mise en œuvre d'une politique de discrimination positive et protectrice permettant aux classes défavorisées d'accéder à l'enseignement supérieur. Avant le début des années 1980, cette expansion de l'enseignement supérieur dépendait essentiellement de la volonté politique et des subsides gouvernementaux. La participation du secteur privé était réduite au strict minimum et obéissait entièrement à des raisons philanthropiques. Jusqu'à cette période, l'enseignement supérieur indien n'avait pas abandonné toutes les caractéristiques de la période coloniale. Tout un pan de l'enseignement supérieur n'avait pour finalité ni l'emploi, ni la recherche. Il se limitait à des cursus ne débouchant pas sur la licence, en arts, sciences et commerce, dans la tradition de l'enseignement libéral qui prévalait alors, sans lien réel avec les exigences économiques et sociales du terrain (Agarwal, 2006a). Au milieu des années 1980, la croissance de l'enseignement supérieur avait ralenti et le pays connaissait les premiers signes d'un grave problème de chômage de sa population diplômée. Le gouvernement de chaque État et le gouvernement fédéral s'étaient également rendu compte du poids que la 
nécessité de maintenir la croissance de l'enseignement supérieur exerçait sur les finances publiques. En 1985, le gouvernement proposa un moratoire sur l'expansion des établissements d'enseignement supérieur général et sur la diversification des filières.

À partir du milieu des années 1980, on confia la gestion de cette expansion aux lois du marché. Le développement de l'enseignement supérieur dans les années 1990 a été largement influencé par les politiques néolibérales qui se sont donné pour objectif de rendre l'économie efficace et compétitive, en réaction à la mondialisation du marché et afin de construire une économie fondée sur le savoir. Les programmes de réforme structurels introduits au début des années 1990 commencèrent à faire sentir leurs effets sur l'économie vers le milieu de la décennie et le pays a adopté une stratégie de développement du secteur des services. La disponibilité d'une main-d'œuvre abondante et rentable disposant d'un bagage universitaire a attiré les compagnies étrangères, et le marché indien de l'emploi a lentement commencé à prendre sa place dans le marché mondial. Le gouvernement et les universités ou les établissements subventionnés n'étaient pas en mesure de répondre à la demande croissante de compétences professionnelles ou techniques. Parallèlement, la classe moyenne se développait et ces familles souhaitaient vivement investir dans l'enseignement professionnel ou technique à cause de sa haute valeur sur le marché de l'emploi. Ainsi, un grand nombre d'établissements privés non subventionnés délivrant un cursus professionnel ou technique furent fondés, principalement au sud et à l'ouest du pays. Depuis lors, le segment de l'enseignement supérieur privé non subventionné n'a cessé de croître de manière soutenue, surtout dans les domaines de l'ingénierie, de la médecine, du management, de la formation des enseignants et des technologies de l'information et de la communication. Aujourd'hui, plus de $80 \%$ des établissements et des effectifs de la filière professionnelle se trouvent dans le secteur privé non subventionné. Les établissements professionnels privés fournissent plus de $86 \%$ des emplois techniques du pays (Kapu et Mehta, 2004). La croissance de l'enseignement supérieur indien trouve ainsi largement son origine dans le marché lui-même et, hormis ce qui entre dans le cadre de l'enseignement scientifique, technologique et des technologies de l'information et de la communication (TIC), aucun effort de planification de la politique ne semble avoir été initié durant ces dix dernières années pour développer d'autres segments de l'enseignement supérieur.

\section{Croissance et disparités}

En termes absolus, la croissance des effectifs a été impressionnante. Elle a progressé d'environ 0,174 million en 1950-51 à 10,48 millions en 2004-05, si l'on fait abstraction de l'enseignement à distance. Cependant, seuls environ $12 \%$ des enfants d'une classe d'âge intègrent l'enseignement supérieur. Même 
si elle dispose du plus grand nombre d'établissements, l'Inde n'a pas été capable de mettre en place un financement qui permette à tous les élèves bacheliers qui le souhaitent de poursuivre des études supérieures. On estime qu'il faudrait pour cela créer au moins trois mille nouvelles universités pouvant accueillir chacune dix mille étudiants (Bhargava, 2006). En effet, le Central Advisory Board on Education (CABE) Committee Report ${ }^{1}$ de 2005 précise clairement qu'aucun pays ne peut se prétendre économiquement avancé si moins de $20 \%$ de ses effectifs scolaires fréquentent l'enseignement supérieur.

\section{Des disparités liées à la géographie, au sexe et à l'origine sociale}

De plus, la croissance des établissements et des effectifs scolaires s'est exercée de façon inégale dans les différents États ${ }^{2}$. Les groupes socio-économiques défavorisés sont également sous-représentés et la participation des filles dans les filières techniques et professionnelles est relativement basse.

On observe de grandes variations géographiques dans le niveau de participation. Le taux brut d'inscription ${ }^{3}$ dans le supérieur pour le pays va de $4,7 \%$ dans le Nagaland à 37,25\% dans le Territoire capital national de Delhi (MHRD, 2006). Il est quatre fois plus élevé dans les zones urbaines que dans les zones rurales.

En ce qui concerne le suivi d'études supérieures, il y a des disparités marquées parmi les différents groupes socio-économiques. Bien que le nombre de filles n'ait cessé de progresser au fil des années, leur part dans les effectifs totaux n'était que de $40 \%$ en 2002-2003. La participation des classes les plus basses, connues sous le nom de Scheduled Castes (SC) ou Scheduled Tribes $(S T)^{4}$ a augmenté elle aussi année après année dans différentes disciplines. Environ $15 \%$ de la population des SC et 3,6\% de celle des ST sont inscrits à différents cursus, chiffres qui les placent encore loin derrière la population générale.

Bien que le taux d'inscription dans le supérieur des filles, des membres des $S C$ et des $S T$ augmente au fil des années, les disparités liées à l'origine sociale et au sexe demeurent un réel sujet d'inquiétude. On peut toutefois faire observer que ces différences dans l'accès à l'enseignement supérieur et au suivi

1. Rapport du comité du bureau directeur du Conseil central de l'éducation.

2. L'Inde (ou plus exactement l'Union indienne) est une fédération divisée en 28 États et 7 Territoires (NdT).

3. GER : gross enrolment ratio.

4. Respectivement «les intouchables» et «les populations aborigènes», faisant l'objet d'une discrimination positive. La Constitution indienne leur accorde un statut spécial. Ces communautés étaient considérées comme extérieures à la société et furent exclues du système du Chaturvarna (division de la société indienne en quatre castes, ou Varnas), qui a été la superstructure sociale de la société indienne dans le sous-continent indien pendant des milliers d'années. Elles ont traditionnellement été reléguées aux emplois les plus bas, sans aucune possibilité de promotion sociale et ont fait l'objet d'exclusion et de discrimination massives. Les membres des SC sont appelés Dalits, ceux des ST, Adivasis (NdT). 
des cours ne font que refléter l'inégalité de l'accès à l'école dans le pays. Sans des interventions et une politique appropriées dans le secteur de l'enseignement scolaire, particulièrement au niveau du collège et du lycée, il ne servira pas à grand-chose de prendre des mesures dans le supérieur pour discriminer positivement les filles et les membres des $S C$ et des $S T$.

\section{Les disciplines}

On peut dire qu'environ $84 \%$ des effectifs se trouvent dans les trois grandes disciplines traditionnelles, à savoir les arts (45\%), les sciences $(20 \%)$ et les sciences humaines (19\%). Les $16 \%$ restants sont répartis dans les différents enseignements professionnels, tels que l'enseignement, l'ingénierie, la technologie, la médecine, l'agriculture, les sciences vétérinaires, le droit, etc. (UGC, 2005). Les inscriptions dans les filières d'ingénierie et de technologie ont représenté $7,5 \%$ des inscriptions totales. Les effectifs dans les disciplines liées à l'agriculture ne représentaient que $0,6 \%$, alors qu'ils étaient négligeables en sciences vétérinaires, avec $0,16 \%$.

Ce déséquilibre au profit des cursus traditionnels montre clairement que le pays continue à se conformer à son héritage colonial, qui mettait l'accent sur un enseignement visant à créer des emplois tertiaires. Bien qu'un pourcentage important de la population se destine à l'agriculture, le nombre d'établissements ainsi que les effectifs de ce secteur sont négligeables. De nombreux jeunes suivent une scolarité normale dans des établissements spécialisés mais ils acquièrent aussi, grâce à ces établissements privés, des compétences supplémentaires qui les aident à trouver des emplois très bien rémunérés dans le secteur privé. Pour que de nouveaux établissements soient créés, la redistribution des programmes disciplinaires doit se faire sur la base d'un examen critique des besoins de l'économie en main-d'œuvre.

\section{Financement et secteur privé}

La part du secteur privé dans le nombre d'institutions d'enseignement supérieur est élevée dans des pays tels que le Chili, le Japon, la Corée du Sud, la Malaisie, le Brésil, les Philippines, le Mexique, la Thaïlande et les États-Unis. Toutefois, ces institutions représentent une proportion relativement faible en termes d'inscrits en Malaisie (39,1\%), au Mexique (33,1\%), en Thaïlande (19,0\%) et aux États-Unis (23,2\%). De même, en Chine, bien que les institutions privées comptent pour $39,1 \%$ des établissements supérieurs, elles ne représentent que $8,9 \%$ des inscrits. Autrement dit, l'enseignement supérieur dans ces pays reste très largement un bien public.

Historiquement parlant, la fonction première de l'État fédéral, en Inde, a été de dispenser et de financer l'enseignement supérieur, ce qui a donné naissance à des établissements dont il était tout à la fois le bailleur de fonds et le propriétaire. Depuis 2001, on observe une augmentation spectaculaire du nombre 
d'établissements d'enseignement supérieur privés et des effectifs dans ces établissements. Le profil général de croissance durant la période 2001-2006 fait apparaître que le nombre d'établissements privés a pratiquement doublé depuis 2001-2002 et que leur part des effectifs durant les cinq années qui vont de 2001 à 2006 a progressé de 76,7\%. En 2000-2001, les établissements privés non subventionnés représentaient $42,6 \%$ du nombre total d'établissements supérieurs, chiffre qui est passé à 63,21\% en 2005-2006 (UGC, 2006). De même, la part des effectifs dans l'enseignement supérieur privé non subventionné est passée de 32,89\% en 2001-2002 à 51,53\% en 2005-2006 (Anandkrishnan, 2006).

À cause du caractère limité des ressources financières et de la priorité donnée à l'enseignement élémentaire, le gouvernement ne pouvait étendre ses subventions aux établissements privés fondés après les années 1980. Ceux-ci fonctionnent à l'aide des frais de scolarité payés par les étudiants et sont appelés "établissements autofinancés ", bien qu'ils doivent se conformer à la réglementation mise en place par le gouvernement et l'université dont ils dépendent.

\section{UNIVERSITÉS ET CHANGEMENTS : DES RÉPONSES AMBIVALENTES}

Alors que les acteurs privés de l'enseignement supérieur ont été suffisamment réactifs pour entamer des réformes structurelles afin de répondre aux exigences de qualification du marché de l'emploi, les établissements publics, eux, ont fait preuve d'une inertie certaine quant à ces mêmes changements structurels, pourtant nécessaires pour adapter leur rôle et leur fonction à un environnement en pleine mutation. Il subsiste un large fossé entre les exigences de qualification de l'économie et le nombre et la qualité des diplômés formés par ces établissements. Les universités, censées être les épicentres de la recherche et de l'innovation, sont restées dans une très large mesure totalement hermétiques au monde des affaires et de l'industrie. Les relations entre le monde universitaire et la communauté nationale sont également plus ambivalentes que jamais.

Alors que les repères autour d'elles changeaient, la position des universités est demeurée ambivalente en ce qui concerne l'inclusion et l'exclusion, les priorités respectives de l'enseignement et de la recherche, les relations qu'elles entretiennent avec l'État et le monde des affaires, les structures de prise de décision, la responsabilité financière et l'autonomie institutionnelle, l'importance et l'obsolescence des disciplines et, plus globalement, en ce qui concerne les changements. Par exemple, il existe une ambivalence profonde à propos de l'autonomie institutionnelle et de la responsabilité financière des universités. L'argument selon lequel il est indispensable de leur assurer une autonomie institutionnelle afin de pouvoir mobiliser des ressources supplémentaires pour définir des buts et des priorités institutionnels contredit la structure de direction actuelle et les pratiques gestionnaires rigides des universités publiques. La 
requête maintes fois formulée par l'université pour obtenir tout à la fois des droits professionnels afin de promouvoir l'enseignement et la recherche et une garantie de liberté d'action dégagée de toute interférence bureaucratique est une contradiction dans les termes, dès lors que les institutions publiques sont frappées d'incapacité ou font preuve de réticence lorsqu'il s'agit de mobiliser des ressources supplémentaires, ou ont une position ambiguë sur l'importance de disciplines à forte valeur ajoutée sur le marché de l'emploi. En fait, il existe une profonde ambivalence en ce qui concerne le rôle que doit jouer l'université pour répondre aux attentes sociales et aux exigences en matière d'aptitudes.

\section{Accès et équité}

Bien que le pays dispose d'accès diversifiés à l'enseignement tertiaire - donnant un statut légal aux établissements privés, mettant en place des structures d'enseignement à distance, introduisant des cursus autofinancés au sein des établissements publics, autorisant quelques prestataires de services étrangers à opérer sur le sol national - de telles stratégies n'ont qu'un impact limité lorsqu'il s'agit de satisfaire la demande d'accès à l'enseignement tertiaire, particulièrement en ce qui concerne les disciplines qui créent des actifs utilisant massivement les différents savoirs. Postérieur aux années 1980, le phénomène d'expansion du secteur non universitaire, et particulièrement des établissements polytechniques et de formation industrielle (gérés essentiellement par le secteur privé), a tenté de satisfaire la demande croissante en matière de formation. Simultanément, se pose avec insistance le problème de la marchandisation des enseignements professionnalisants dispensés par le secteur privé, qui va à l'encontre du principe d'égalité d'accès à l'éducation. Autoriser le secteur privé à compléter les efforts de l'État pour étendre le système d'enseignement supérieur revient d'une certaine manière à institutionnaliser l'exclusion, et tout particulièrement celle qui touche l'accès à des filières très porteuses. Bien que l'État fédéral n'ait pas ménagé ses efforts pour protéger les intérêts des groupes défavorisés en introduisant des mesures de discrimination positive telles que la politique de réservation, les bourses, les prêts aux étudiants, etc., les interventions extérieures destinées à arrondir les budgets ne manquent pas. Créer les conditions qui permettent aux personnes socio-économiquement défavorisées d'accéder en plus grand nombre à l'enseignement supérieur est une préoccupation de tous les instants.

\section{Réglementation et assurance qualité}

L'enseignement supérieur en Inde est assuré à la fois par des universités et des établissements spécialisés, entre lesquels il existe une relation symbiotique. Alors que l'enseignement et l'apprentissage constituent un point commun, seules les universités délivrent des licences. Environ $85 \%$ de l'enseignement 
supérieur sont assurés par des établissements spécialisés délivrant un enseignement antérieur et postérieur au diplôme auquel ils préparent (au nombre de $17625)$. Ils sont affiliés à une université, dont ils dépendent.

Il existe trois façons de fonder une université en Inde : par une loi émanant du gouvernement fédéral, par une loi émanant de l'un des États ou enfin par un ordre exécutif du gouvernement fédéral après recommandation de $l^{\prime} U G C^{5}$. En fonction du mode de fondation, ces universités entrent grosso modo dans trois catégories : les universités fédérales, les universités d'État et les universités accréditées ${ }^{6}$. Les universités disposent d'une autonomie pédagogique appréciable, alors que les établissements spécialisés ont une marge de liberté fort réduite, ne laissant guère de place à l'innovation. Ils fonctionnent comme des entités mécaniquement régies par les universités.

Traditionnellement, la pérennité des normes en matière d'enseignement supérieur a toujours été du ressort du gouvernement fédéral. À l'origine, ce rôle était confié à l'UGC mais, au fil du temps, le gouvernement fédéral a mis en place jusqu'à vingt-cinq Conseils, dont certains sont des entités statutaires, pour établir des normes et des référentiels sous forme de règlements et de recommandations régissant différents domaines d'étude et pour mettre ceux-ci en œuvre, afin d'assurer la qualité de l'enseignement et la pérennité des exigences.

Ces Conseils servent également de réseau nerveux entre le gouvernement fédéral et ceux des différents États ainsi qu'entre les établissements d'enseignement supérieur. De plus, le gouvernement fédéral a également créé deux organisations indépendantes au niveau national, le National Assessment and Accreditation Council ${ }^{7}$ (NAAC) et le National Board of Accreditation ${ }^{8}$ (NBA), afin d'accréditer établissements et programmes. Le $N A A C$ a reçu pour mission l'évaluation des performances et des résultats des universités et des établissements spécialisés, ainsi que leur accréditation. L'idée sous-jacente est de permettre aux établissements de maximaliser leurs ressources et leur potentiel en les informant de leurs forces et de leurs faiblesses. À ce jour, la NAAC n'a accrédité que 131 des 380 universités et 3074 établissements spécialisés sur environ 17000 .

Au vu de l'organisation actuelle, il est évident qu'il existe de multiples instances de réglementation, qui semblent être aux prises avec des problèmes de coordination et de chevauchement de compétences. Alors que chacune de ces instances a dûment fait la preuve de son utilité, c'est bien l'absence d'une structure coordinatrice et fédératrice qui pose problème.

5. Commission des attributions universitaires.

6. "Deemed to be Universities », c'est-à-dire une structure jugée digne d'être une université (NdT).

7. Conseil national d'évaluation et d'accréditation.

8. Bureau national d'accréditation. 


\section{L’internationalisation}

Il existe également un autre problème, lié à la prolifération des établissements étrangers. En Inde aussi, les universités étrangères ont commencé à collaborer avec les établissements privés pour transmettre l'enseignement et décerner des diplômes universitaires aux étudiants. Les étudiants indiens étant très friands de diplômes étrangers, les tendances établies par le marché seront la norme dans l'avenir. Quelques universités indiennes, comme la Manipal Academy of Higher Education (MAHE), le Birla Institute of Technology and Science (BITS) et l'Indira Gandhi National Open University (IGNOU) ont également créé des campus à l'étranger. D'une certaine manière, l'internationalisation de l'enseignement supérieur est une tendance inévitable. Cependant, le pays doit encore préciser sa politique en matière de réglementation relative à l'entrée sur le territoire national d'établissements d'enseignement étrangers. Certains problèmes afférents, tels que la réglementation d'entrée et de fonctionnement, la pérennisation de la qualité et la lutte contre la marchandisation, doivent être décantés et réglés.

\section{Autonomie et responsabilité financière}

Durant la période coloniale, les universités indiennes ont pris modèle sur les universités britanniques, ce qui a donné naissance au système d'affiliation de l'enseignement supérieur indien.

Toutefois, les universités et les établissements supérieurs spécialisés indiens ne jouissent pas du genre d'autonomie administrative et financière qui existe dans les pays développés. Pour des raisons que l'on peut comprendre, le système s'est montré particulièrement réticent à déléguer ces pouvoirs à des unités décentralisées, qu'il s'agisse d'universités ou d'établissements spécialisés. Plusieurs comités et commissions mis en place dans le passé n'ont cessé de répéter que l'autonomie administrative et financière était une condition essentielle dans la quête de l'excellence universitaire.

\section{Bien public, bien privé?}

L'enseignement supérieur est généralement reconnu comme étant un «bien public», ou du moins, « un bien quasi-public» (CABE, 2005a : p. 7). Compte tenu de la structure même de l'enseignement supérieur dans le pays, les États étaient les seuls et uniques responsables du financement et de l'administration des établissements d’État jusqu'au milieu des années 1970. Le gouvernement fédéral était responsable du financement et de l'administration des universités fédérales et des établissements d'importance nationale. Il était en outre responsable de la promotion des études professionnelles, scientifiques et spéciales, ainsi que de celle de la formation continue. Dans l'ensemble, le 
financement demeure de la responsabilité des États. Cependant, à cause de différents facteurs parmi lesquels figurent les nouvelles politiques économiques adoptées depuis les années 1990, la participation actuelle de l'État dans l'enseignement en général, et dans l'enseignement supérieur en particulier, décroît sur le terrain et dans les faits. La part du gouvernement fédéral dans ce financement tourne autour de $20 \%$ depuis 1990-1991, à quelques exceptions près dans les années 2000, où elle est montée à un peu plus de $25 \%$.

La Commission de l'enseignement (1964-1966) s'était attelée à une mission exhaustive afin d'évaluer les besoins en ressources et avait recommandé d'augmenter l'enveloppe budgétaire de l'éducation jusqu'à atteindre au moins $6 \%$ du PNB à l'horizon 1986. Toutes les expertises ont depuis réitéré cette recommandation. Malheureusement, cette promesse demeure un rêve inaccessible. La part du PNB allouée à l'éducation (qui regroupe les recettes et les capitaux) est passée du chiffre extraordinairement bas de 0,67\% en 1951-1952 à un maximum historique de 4,4\% en 2000-2001. Depuis, toutefois, ce pourcentage est retombé brutalement à 3,54\% en 2004-2005 (MHRD, 2005). Ainsi, la priorité accordée à l'éducation n'a cessé de décliner depuis 1990, si l'on fait abstraction de quelques années autour de 2000-2001. De plus, à partir du milieu des années 1980, la priorité budgétaire a été l'enseignement élémentaire, tout spécialement après la mise en place de la Politique nationale d'éducation (1986).

Dans ce contexte d'allocation de ressources intra-sectorielle, on a pu observer que l'engagement constitutionnel de fournir un enseignement élémentaire universel n'était pas négociable. Mais l'enseignement secondaire ne peut être ignoré, qu'il soit considéré comme une porte d'entrée vers des études supérieures ou comme un enseignement terminal. Enfin, dans un contexte de mondialisation et de concurrence exacerbée, l'enseignement supérieur ne peut lui non plus être négligé. Eu égard à ces réalités, une espèce de consensus émerge progressivement pour accorder au moins $3 \%$ du PNB à l'enseignement élémentaire, $1,5 \%$ à l'enseignement secondaire et le 1,5\% restant aux enseignements supérieur et technique (CABE, 2005a, p. 46; MHRD, 1999 \& 2005, p. 12).

Depuis les années 1990, la priorité donnée aux enseignements supérieur et technique s'est estompée. Mises bout à bout, les allocations attribuées à ces enseignements constituaient à peine 0,6\% du PNB en 1990-1991, pour descendre à 0,46\% en 2004-2005 (BE).

$\mathrm{Au}$ fil des années, les sources de financement de l'enseignement supérieur se sont diversifiées pour faire face à cette tendance au désengagement des dépenses publiques. La tendance générale a été de faire porter l'effort financier de l'enseignement supérieur par les bénéficiaires directs - les étudiants - et les utilisateurs finaux - les employeurs. Durant les années 1990, quelques-unes des réformes auxquelles les pays en voie de développement ont dû se résoudre ont inclus le fait d'encourager une plus grande différenciation au sein des établissements d'enseignement supérieur - y compris le développement des 
établissements privés -, l'introduction de mécanismes de recouvrement comprenant une redistribution des coûts de fonctionnement auprès des étudiants, la modification du secteur de l'enseignement supérieur pour le rendre plus réceptif aux lois du marché et pour accorder aux établissements publics davantage d'autonomie, et enfin d'encourager la priorité donnée aux investissements sur les mécanismes d'amélioration de la qualité (Banque mondiale, 1994). En Inde, les réformes liées à la mobilisation des ressources pour réduire les dépenses publiques ont inclus la promotion de l'enseignement à distance, l'encouragement du secteur privé et des méthodes de recouvrement des coûts, méthodes qui vont de pair avec une réduction des subventions allouées à l'enseignement supérieur.

Diverses mesures de diversification des recettes ont inclus une augmentation des frais d'inscription et de scolarité des étudiants, la mise en place de programmes de prêts étudiants gérés par des banques commerciales, et la privatisation. Différentes mesures de collecte de fonds ont été appliquées, telles que la hausse des frais de scolarité comme source significative de revenus pour subvenir aux coûts de la scolarité, le recouvrement total des frais induits par d'autres prestations payantes (comme la pension complète fournie par l'établissement), la vente de publications de recherche, la mise en place d'audits, etc.

L'enseignement supérieur de qualité demeure par nature élitiste, puisqu'il n'est toujours pas accessible à l'immense majorité de la population qui devrait pouvoir en bénéficier. La faute en incombe avant tout au changement de politique qui s'est produit au milieu des années 1980, qui a anticipé la croissance des établissements publics et qui a laissé un marché largement ouvert aux établissements privés offrant des cursus fortement rémunérateurs. On peut objecter que cette poussée significative des établissements privés équivaut de fait à une marchandisation de l'enseignement supérieur (Tilak, 2006), ce qui ne manque pas de poser des questions éthiques d'accès aux études et d'équité.

\section{BIBLIOGRAPHIE}

AGARWAL Pawan (2006a): Higher Education and the Labour Market in India. Indian Council for Research on International Economic Relations (ICRIER), New Delhi. worldbank.org/INTABCDE2007BEI/Resources/PAgarwal.PDF.

ANANDKRISHNAN M. (2006): "Privatization of Higher Education: Opportunities and Anomalies". Communication présentée dans le cadre du séminaire national 'Privatization and Commercialization of Higher Education', organisé par le NIEPA, New Delhi, 2 mai 2006, mimeo. 
BHARGAVA P. (2006): "Knowledge and National Development". Communication présentée dans le cadre du séminaire national 'Education Commission' organisé par le NUEPA, New Delhi, 26-28 décembre 2006, mimeo.

CARNOY Martin (2006): Higher Education and Economic Development: India and China and the $21^{\text {st }}$ Century, Working Paper No. 297, Stanford Center for International Development.

KAPU Devesh and MEHTA P. B. (2004): Indian Higher Education Reform: From Half-Baked Socialism to Half-Baked Capitalism, CID Working Paper No. 108, Center for International Development at Harvard University.

MHRD, Government of India (2005): CABE Committee Report on Autonomy of Higher Education Institutions, June 2005.

Ministry of Human Resource Development (2005): Report of the Committee on National Common Minimum Programme's Commitment of Six Per Cent of GDP to Education, NIEPA, New Delhi.

Ministry of Human Resource Development (Various Years): Analysis of Budgeted Expenditure on Education, MHRD, New Delhi.

Ministry of Human Resource Development (Various Years): Selected Educational Statistics, MHRD, New Delhi.

PATEL I. G. (2003) : "Higher education and Economic Development", in J.B.G. Tilak (ed.) Education, Society and Development: National and International Perspectives. New Delhi : APH for NIEPA.

PRAKASH Ved (2007): "Trends in Growth and Financing of Higher Education in India". Seminar on Higher Education for Growth and Equity: India-China Experience, 20-21 janvier 2007.

TILAK J.B.G. (2006) : "Private Higher Education: Philanthropy to Profits" in Higher Education in the World 2006: The Financing of Universities, GUNI Series on the Social Commitment of Universities 1, Palgrave, New York and GUNI.

University Grants Commission (UGC) (Various Years): Annual Reports, UGC, New Delhi. 